\title{
Optimizing Follow-up Assessment of Patients with Cutaneous Melanoma
}

\author{
Neal Bhutiani ${ }^{1}$, Michael E. Egger ${ }^{2}$, and Kelly M. McMasters ${ }^{1}$ \\ ${ }^{1}$ Division of Surgical Oncology, The Hiram C. Polk, Jr., MD Department of Surgery, University of Louisville School of \\ Medicine, Louisville, KY; ${ }^{2}$ Department of Surgical Oncology, University of Texas MD Anderson Cancer Center, Houston, \\ TX
}

Recently, the use of imaging studies for follow-up assessment of melanoma patients has become a topic of significant controversy. For most of the last 50 years, the prevailing wisdom within the oncology community has been that the majority of melanoma recurrences are detectable by patients or physicians. As a result, the current National Comprehensive Cancer Network (NCCN) guidelines provide wide latitude regarding imaging, allowing physicians to "consider" imaging at intervals ranging from every 3 to 12 months or not at all, depending on the stage and substage of the disease. ${ }^{1}$ The unspoken reality enabling this highly variable use of imaging studies was that early detection of stage 4 disease offered little value because we had no effective treatments (except perhaps for some patients with resectable metastatic disease). In fact, some might argue that many therapies used for advanced melanoma during the past several decades have been worse than the disease, conferring significant toxicity and little, if any, benefit. In that context, neither physicians nor patients were disadvantaged by waiting for recurrences to become symptomatic or detectable on physical examination.

Times have changed. Widely available Food and Drug Administration (FDA)-approved immune checkpoint inhibitors currently offer the hope of durable complete remission-even cure-for a reproducible fraction of patients with advanced melanoma. Adoptive cellular therapy offers similar hope at centers in which such treatment is available. Although early detection of recurrent

(C) Society of Surgical Oncology 2017

First Received: 2 January 2017;

Published Online: 24 January 2017

K. M. McMasters

e-mail: mcmasters@louisville.edu melanoma has not been shown to improve survival to date, an inverse relationship between disease burden and response to immunotherapy is generally acknowledged. Thus, a reasonable presumptive rationale exists for early detection of asymptomatic metastasis. Every oncologist ostensibly prefers to treat a patient with isolated lung metastases rather than lung and brain metastasis.

The work of Lee and colleagues from Memorial Sloan Kettering Cancer Center (MSKCC), patterns and timing of initial relapse in pathologic stage II melanoma patients, continues the effort started by Romano et al. ${ }^{2}$ in 2010 , who assessed patterns of follow-up assessment for stage 3 disease and used those findings to make recommendations regarding follow-up evaluation. The current article addresses the very salient question regarding recurrence in the heterogeneous stage 2 disease population. The authors' findings continue to validate the American Joint Committee on Cancer (AJCC) stage subgroupings, namely, that disease stages $2 \mathrm{~A}, \mathrm{~B}$, and $\mathrm{C}$ behave very differently in terms of rates and patterns of recurrence. However, they nicely contextualize those differences in a way that potentially directs a follow-up strategy.

The authors define a rational follow-up strategy by delineating the time course and location of recurrences according to substage and by pairing those recurrences with the methods of detection. Interestingly, the most common method for detecting recurrence is detection by the patients themselves. Of course, this was a somewhat self-fulfilling prophesy in this retrospective analysis because no standard imaging protocols were followed, and imaging was generally considered to be optional by NCCN guidelines. It remains unknown how many patients might have had asymptomatic recurrence detected by imaging had it been used routinely. Nevertheless, the authors' study points out one of the constant, repeatedly validated truths 
in medicine: a thorough history and physical examination directed at detecting signs and symptoms of recurrent disease remain the mainstay of follow-up assessment. Imaging, frankly, is not a substitute for physical examination, and the embarrassing truth of the matter is that many cancer specialists never ask melanoma patients to disrobe for a proper physical examination.

Sherry and his colleagues at the National Cancer Institute (NCI) analyzed data across multiple clinical trials of immunotherapy for high-risk stages 2, 3, and 4 melanoma patients. The difference between this study and the investigation from MSKCC was that routine surveillance computed tomography (CT) imaging was prescribed in the NCI clinical trials. Sherry and colleagues found that for stage 2 patients, the first site of recurrence was locoregional half the time and systemic the other half. One third of stage 3 patients had locoregional recurrence, and two thirds had systemic recurrence. The use of CT scans detected $59 \%$ of recurrences overall and $75 \%$ of the patients with asymptomatic systemic recurrences. Laboratory studies were essentially useless.

Both of these studies begin to explore the more provocative underlying question: how should we tailor the follow-up plan, including the use of surveillance imaging studies, to match the risk of recurrence? Although the AJCC staging system is the obvious starting point for discerning differences in prognosis, we submit that anatomic staging uses technology that is hundreds to thousands of years old, namely, what a physician can see or feel and what a pathologist can visualize under the microscope. Nonetheless, clinicopathologic factors such as Breslow thickness, ulceration, and sentinel lymph node status provide invaluable prognostic information.

However, TNM staging carries significant limitations. For example, every patient with a small focus of metastasis in a sentinel lymph node is considered stage 3 , yet the 5-year survival rate ranges from 15 to $85 \%$ depending on other factors. Prognostic models that incorporate other factors such as age, anatomic location of the primary tumor, and non-sentinel node metastasis can provide better estimates of prognosis than the AJCC stage alone.

Whether a physician chooses to use the AJCC's online model, ${ }^{3}$ available at www.melanomaprognosis.net, the model developed at the University of Louisville at www. melanomacalculator.com (also available as a free iPhone or Android app), ${ }^{4}$ or another model, building a rational follow-up plan begins with an individualized assessment of recurrence risk. The future of optimizing this risk assessment clearly lies in genetic and molecular profiling. A validated polymerase chain reaction (PCR)-based multigene assay for cutaneous melanoma is available, ${ }^{5,6}$ although how such a test should influence a follow-up plan-alone or in conjunction with clinicopathologic factor models-remains unclear and warrants further investigation.

Although it remains to be seen whether earlier detection of melanoma recurrence will improve survival in the era of effective immunotherapy for melanoma, development of a rational, standardized follow-up system tailored to individual patient risk seems to be an appropriate step in the right direction. Critics will point to the cost of imaging and demand cost-effectiveness studies. However, we argue that melanoma represents virtually the only cancer in which patients can have a $50 \%$ or greater risk of recurrence, and the NCCN guidelines allow for optional imaging.

How will we pay for it? We suggest that we start by eliminating all 3-6 month laboratory and imaging tests to detect asymptomatic recurrence of patients with cancers for which no effective salvage therapies exist. Physicians reflexively and routinely order such studies for follow-up of patients with pancreatic cancer, extrahepatic cholangiocarcinoma, and gastric and esophageal adenocarcinoma, although we know that systemic therapy for recurrent disease offers little benefit. In fact, it can be argued that the benefit for these patients is defined by Q-TWiST (Quality Time WIthout Symptoms or Toxicity-knowledge of recurrence). We certainly rob many such patients of the time without knowledge of recurrence to begin systemic therapy for asymptomatic metastasis, which often promises limited or no real benefit in terms of quantity or quality of life. However, in the case of advanced melanoma, we currently have effective therapies that can improve survival and offer the hope of cure. For potential maximization of that promise, should we not strongly consider personalized follow-up plans to match the risk of recurrence? The new era of melanoma treatment demands new surveillance strategies.

\section{REFERENCES}

1. National Comprehensive Cancer Network (NCCN). In: NCCN clinical practice guidelines in oncology. 2016. http://www.ncen. org/professionals/physician_gls/f_guidelines.asp. Accessed 15 Dec 2016.

2. Romano E, Scordo M, Dusza SW, et al. Site and timing of first relapse in stage III melanoma patients: implications for follow-up guidelines. J Clin Oncol. 2010;28:3042.

3. Soong SJ, Ding S, Coit D, Balch CM, Gershenwald JE, Thompson JF, Gimotty P, AJCC Melanoma Task Force. Predicting survival outcome of localized melanoma: an electronic prediction tool based on the AJCC Melanoma Database. Ann Surg Oncol. 2010;17:2006-14.

4. Callender GG, Gershenwald JE, Egger ME, Scoggins CR, Martin RC II, Schacherer CW, et al. A novel and accurate computer model of melanoma prognosis for patients staged by sentinel lymph node biopsy: comparison with the American Joint Committee on Cancer model. J Am Coll Surg. 2012;214:608-17. 
5. Gerami P, Cook RW, Wilkinson J, Russell MC, Dhillon N, Amaria $\mathrm{RN}$, et al. Development of a prognostic genetic signature to predict the metastatic risk associated with cutaneous melanoma. Clin Cancer Res. 2015;21:175-83. doi:10.1158/1078-0432.CCR-133316.
6. Gerami P, Cook RW, Russell MC, Wilkinson J, Amaria RN, Gonzalez R, et al. Gene expression profiling for molecular staging of cutaneous melanoma in patients undergoing sentinel lymph node biopsy. J Am Acad Dermatol. 2015;72:780-5.e3. 